\title{
Peroxiredoxin proteins protect MCF-7 breast cancer cells from doxorubicin-induced toxicity
}

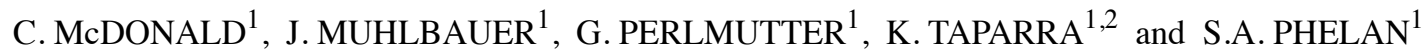 \\ ${ }^{1}$ Fairfield University, Fairfield, CT 06824; ${ }^{2}$ Johns Hopkins School of Medicine, \\ Cellular and Molecular Medicine, Baltimore, MD 21205, USA
}

Received February 28, 2014; Accepted April 2, 2014

DOI: $10.3892 /$ ijo.2014.2398

\begin{abstract}
Peroxiredoxin (Prdx) proteins are thiol-specific antioxidants that protect cells from oxidative stress in many normal and disease states. There are six Prdx proteins expressed in mammals, each with a characteristic tissue expression, subcellular distribution and substrate specificity. Recent studies have revealed elevated Prdx levels in many cancers, suggesting a protective role for these proteins in cancer cell survival. The present study is the first to investigate the function of all six Prdx proteins in the MCF-7 breast cancer cell line. We show that these cells have both higher resistance to doxorubicin-induced toxicity and significantly elevated Prdx levels, compared to the non-cancer MCF-10A cells. Using transient siRNA transfections, we show that Prdx3 suppression leads to decreased MCF-7 cell survival in the absence of doxorubicin. We further demonstrate that individual suppression of four of six of the Prdx proteins leads to increased doxorubicin-induced toxicity by apoptosis. Finally, we show that clonal selection of a doxorubicin-resistant MCF-7 subline by 2 -week culture in $0.1 \mu \mathrm{M}$ doxorubicin resulted in a marked elevation in the expression of several Prdx proteins. Together, these data reveal a protective function for peroxiredoxins in MCF-7 cell survival, and suggest that Prdx overexpression in breast cancer may play a role in doxorubicin-resistance in these, and possibly other, breast cancer cells. This study is the first to investigate the function of the entire Prdx family in a breast cancer cell line.
\end{abstract}

\section{Introduction}

The peroxiredoxins (Prdxs) are a ubiquitous family of evolutionarily conserved antioxidant proteins that reduce aqueous and lipid peroxides associated with normal

Correspondence to: Dr Shelley A. Phelan, Fairfield University, 1073 North Benson Road, Fairfield, CT 06824, USA

E-mail: sphelan@fairfield.edu

Key words: peroxiredoxins, antioxidants, MCF-7, breast cancer, cell death, doxorubicin metabolism (1-3). There are six members of the mammalian peroxiredoxin family, which can be subdivided into three classes: the 2-Cys Prdxs (Prdx1-4), the atypical 2-Cys Prdx $(\operatorname{Prdx} 5)$ and the 1-Cys $\operatorname{Prdx}(\operatorname{Prdx} 6)$. These proteins reduce cellular substrates by converting their active-site cysteines to sulfenic acid, which can be re-reduced by thiols such as ascorbic acid or glutathione through different mechanisms for different classes. These proteins are highly abundant in mammalian cells, suggesting an important role in antioxidant defense. In addition, peroxiredoxins participate in redox-sensitive signal transduction pathways, and are know to have effects on cell growth, proliferation, differentiation and apoptosis $(4,5)$.

It has long been recognized that cancer cells harbor elevated levels of highly reactive oxygen species (ROS) such as hydrogen peroxide and superoxide $(6,7)$, exhibiting many signs of an oxidatively stressed environment. There is evidence that this oxidative stress may be a factor in both cancer initiation and/or cancer progression, depending on the tumor (8). Unlike normal cells, which are susceptible to cytotoxicity from such high levels of ROS, cancer cells are relatively resistant and can evade cell death, yet the precise mechanisms are not clear. Over the past several years, studies have reported overexpression of Prdxs in several types of cancer (9-14), and there is mounting evidence that Prdxs play a role in carcinogenesis $(9,15-19)$. These data suggest that cancer cell resistance to ROS may be provided, at least in part, through peroxiredoxin overexpression, leading to increased antioxidant activity and/or alteration in key redoxregulated growth and death pathways. However, there is very little understanding of the precise role peroxiredoxins play in this protection or the mechanism that governs increased peroxiredoxin expression in cancer cells.

Many studies have reported that breast tumors exhibit elevated levels of Prdxs compared to normal breast epithelial tissue $(10,12,16,20)$. Furthermore, based on evidence that an adaptive oxidative stress response is critical to chemoresistance, it was recently suggested that peroxiredoxins are likely to be key players in chemoresistance in breast cancer, and may be potential targets for intervention (21). While clinical studies are important, a comparison of relevant cell lines can be a valuable tool in understanding the role of Prdxs in breast cancer biology and drug resistance. We and others have shown significant overexpression of Prdxs in the MCF-7 
adenocarcinoma cell line, as compared to the non-cancerous MCF-10A breast epithelial line (22-24). We previously demonstrated that MCF-7 cells are much more resistant to $\mathrm{H}_{2} \mathrm{O}_{2}$-induced apoptosis than the non-malignant MCF-10A breast cells (22). Bae et al also reported elevated Prdx levels in MCF-7 cells, and went on to show that overexpression of either Prdx 1 or Prdx 2 in MCF-10A cells conferred resistance to $\mathrm{H}_{2} \mathrm{O}_{2}$-induced apoptosis (22). Furthermore, radiationresistant lines derived from the MCF-7 cells have elevated Prdx 2 levels, and Prdx2 suppression in these cells partially reversed the radiation resistance (25). Taken together, these studies suggest a protective role for Prdxs in breast cancer and suggest that use of these cell lines may be an important tool in understanding the function of Prdxs in breast cancer.

Based on these data and implications, we sought to examine the role of Prdxs in MCF-7 cell survival and doxorubicin-resistance using siRNA-mediated protein suppression. Doxorubicin is an anthracycline antibiotic that has been used as an effective chemotherapy agent in the treatment of breast cancer in patients, although many patients develop resistance to the drug leading to aggressive relapse (26). Doxorubicin induces oxidative stress and apoptosis in MCF-7 cells (27), suggesting that antioxidants may be an important line of defense in this and other breast cancer cells. We hypothesized that Prdx suppression in MCF-7 cells would decrease the viability of these cells and increase their susceptibility to doxorubicin-induced toxicity. Likewise, we hypothesized that prolonged exposure of MCF-7 cells to doxorubicin would lead to induction of Prdx expression.

\section{Materials and methods}

Cell culture. MCF-7 cells were cultured in ATCC-formulated Eagle's minimum essential medium containing bovine insulin $(0.01 \mathrm{mg} / \mathrm{ml})$ and $10 \%$ fetal bovine serum. MCF-10A cells were cultured in MEBM medium, supplemented with BPE $(13 \mathrm{mg} / \mathrm{ml})$, hydrocortisone $(0.5 \mathrm{mg} / \mathrm{ml}), \mathrm{hEGF}(10 \mu \mathrm{g} / \mathrm{ml})$, insulin $(5 \mathrm{mg} / \mathrm{ml})$, and cholera toxin $(100 \mathrm{ng} / \mathrm{ml})$. Both cell lines were cultured at $37^{\circ} \mathrm{C}$ in a humidified $5 \% \mathrm{CO}_{2}$ atmosphere.

Doxorubicin treatments. To determine resistance to doxorubicin, MCF-7 and MCF10-A cells were cultured in 48-well plates and treated the following day with 0.1 or $0.5 \mu \mathrm{M}$ doxorubicin for $48 \mathrm{~h}$. To determine the effect of Prdx suppression on doxorubicin-resistance, MCF-7 cells were allowed to grow for $48 \mathrm{~h}$ after siRNA transfection and then were treated with $0.5 \mu \mathrm{M}$ doxorubicin for an additional $24 \mathrm{~h}$. For generation of a doxorubicin-resistant culture, MCF-7 cells were subcultured for 14 days in T-75 flasks in the presence of $0.1 \mu \mathrm{M}$ doxorubicin, with media and treatment replacement every 3-4 days.

Measurement of viable cell density. Cell viability was determined using an MTT 3-(4,5-dimethylthiazol-2-yl)-2,5-diphenyltetrazolium bromide assay. MCF-7 and MCF-10A cells were seeded into separate 48-well plates in replicate groups of four and transfection/treatment experiments conducted. After treatment incubation, cells were assayed for MTT at a confluency less than $80 \%$. Cells were rinsed with phenol redfree medium and incubated with $0.5 \mathrm{mg} / \mathrm{ml} \mathrm{MTT} \mathrm{(diluted} \mathrm{in}$ phenol red-free medium) for $1.5 \mathrm{~h}$ at $37^{\circ} \mathrm{C}$. This medium was then removed and replaced with $200 \mu \mathrm{l}$ of acidic isopropanol and the plate was rocked for $5 \mathrm{~min}$ at room temperature. The absorbance of the solubilized product was read at $570 \mathrm{~nm}$ using the corresponding absorbance of cell-free wells or the absorbance at 650 for background subtraction.

Suppression of Prdx1-6 by siRNA. MCF-7 cells were seeded into 24-well plates at 50,000 cells per well. Twenty-four hours after seeding, cells were transfected using Silencer Select siRNA (Ambion, Austin, TX, USA) for Prdx1-6 (or a negative control siRNA) at a final concentration of $33 \mathrm{nM}$ using the Lipofectamine 2000 reagent (Invitrogen, Carlsbad, CA, USA). The siRNA ID\#s are as follows: Prdx1 (s10007), Prdx2 (s13959), Prdx3 (s21507), Prdx4 (s20686), Prdx5 (s24559), and Prdx6 (s18429). Briefly, for each well 20 pmoles of siRNA was mixed with $1 \mu \mathrm{l}$ Lipofectamine 2000 reagent and $98 \mu \mathrm{l}$ Opti-MEM I serum-free media (Invitrogen), allowed to precipitate for $20 \mathrm{~min}$, and subsequently added to wells. Transfected cells were cultured for $48 \mathrm{~h}$ followed by protein extraction and western blot analysis to determine levels of Prdx suppression. Transfected cells used for cell viability and toxicity assays were treated with or without doxorubicin for an additional $24 \mathrm{~h}$ prior to end-point assays.

Western blot analysis. For protein analysis, cells were lysed in mammalian protein extraction reagent (MPER) (Thermo Scientific, Waltham, MA, USA) according to the product suggestions. Protein was quantified using the Coomassie Blue Protein Assay Reagent (Bio-Rad, Hercules, CA, USA), and lysates were separated on a $12 \%$ Mini Protean TGX gel and electrophoretically transferred on to an ImmunBlot PVDF membrane (Bio-Rad). Blots were blocked and incubated with primary antibodies from Abcam [anti-Prdx1 (ab59538), antiPrdx2 (ab15572), anti-Prdx3 (ab16751), anti-Prdx4 (ab59542), anti-Prdx 5 (ab16944) anti-Prdx6 (ab16947); Cambridge, MA, USA]. An antibody for GAPDH (Sigma, St. Louis, MO, USA) was used as a loading control. Blots were subsequently processed with the appropriate secondary antibody and chemiluminescent CDP-Star Reagent, and imaged with X-OMAT film (Kodak). Bands were quantified using Image $\mathbf{J}$ software.

Measurement of cytotoxicity. Cytotoxicity was determined by the indirect measurement of lactate dehydrogenase (LDH) activity using the Cytotox 96 Assay (Promega, Madison, WI, USA). Cells were transfected as described above and cultured for $48 \mathrm{~h}$. To measure released $\mathrm{LDH}$, cell medium was removed and $40 \mu \mathrm{l}$ was assayed using an equal volume of substrate mix and processed according to manufacturer's recommendations. Absorbance was measured at $490 \mathrm{~nm}$ and absorbance of media blanks (with no cells) was subtracted from each value.

Measurement of apoptosis. A membrane permeability/ dead cell apoptosis kit (Invitrogen) and Hoechst 33342 (Life Technologies) were used to detect cell death. Cells were transfected according to the previous methods and treated with or without $0.5 \mu \mathrm{M}$ doxorubicin for $24 \mathrm{~h}$. Cells were stained with $1 \mu \mathrm{l} / \mathrm{ml}$ Yo-Pro and $1 \mu \mathrm{g} / \mathrm{ml}$ Hoechst dye and photographed using phase contrast and fluorescence microscopy. The field of view was quantified as a percent of cells staining positive for Hoechst and Yo-Pro. 


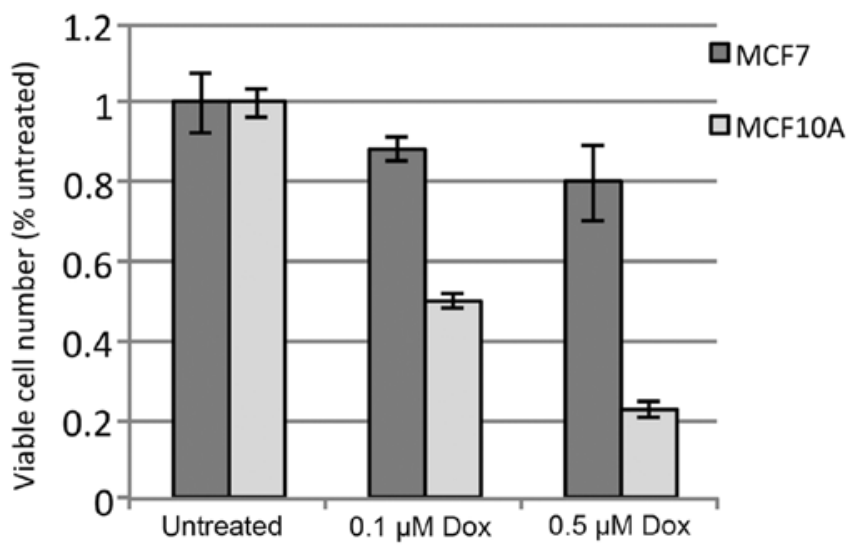

Figure 1. MCF-7 cells are relatively resistant to doxorubicin-induced toxicity. MCF-7 and MCF-10A cells were cultured under normal growth conditions and seeded into the well plates. Cells were treated for $48 \mathrm{~h}$ with $0,0.1$ or $0.5 \mu \mathrm{M}$ doxorubicin and viable cells quantified using the MTT assay. An average from six replicates for each treatment $( \pm \mathrm{SD})$ is shown. $(\mathrm{p}<0.005$ for MCF-10A treatments vs. control; $\mathrm{p}<0.05$ for MCF-7 treatments vs. control.)

Statistical analysis. The means of individual treatment groups in each quantitative experiment were statistically compared using a two-tailed Student's t-test, assuming equal variances.

\section{Results}

We first compared the non-cancerous MCF-10A cell line with the MCF-7 breast adenocarcinoma for sensitivity to doxorubicin-induced toxicity. As shown in Fig. 1, MCF-10A cells exhibited an approximately $50 \%$ reduction in viable cells after $24 \mathrm{~h}$ with $0.1 \mu \mathrm{M}$ doxorubicin, as compared to untreated cells, and a nearly $80 \%$ reduction with $0.5 \mu \mathrm{M}$ doxorubicin. In contrast, MCF-7 cells exhibited only a 10 and $20 \%$ reduction in viability with the same treatments, respectively. The data show significant tolerance of the MCF-7 cell line to this drug treatment. Analysis of peroxiredoxin protein expression in these two lines revealed that expression of five of the six Prdx proteins (Prdx1-5) are markedly elevated in MCF-7 cells, as compared to MCF-10A cells (Fig. 2). Together, these data show a correlation between doxorubicin resistance and peroxiredoxin expression in MCF-7 cells.

In order to address the potential role of Prdxs in $\mathrm{MCF}-7$ cell survival, we used transient siRNA transfection experiments to suppress individual Prdx proteins in these cells. Cells were transfected with $33 \mathrm{nM}$ siRNA and Prdx levels measured after $48 \mathrm{~h}$ by western blot analysis. As shown in Fig. 3A, we were able to greatly reduce the expression of all six Prdx proteins by this method. Quantification of these levels is shown in Fig. 3B, which demonstrates a range between 70 and $90 \%$ protein suppression relative to cells transfected with a negative control siRNA.

Before examining the effect of Prdx suppression on doxorubicin sensitivity, we determined whether Prdx suppression in these cells affected their morphology or viability. Seventytwo hours after transfection (and $24 \mathrm{~h}$ after suppression was measured by western blot analysis) the cells were examined by phase contrast microscopy and analyzed for cytotoxicity using the released LDH assay. First, we found no difference

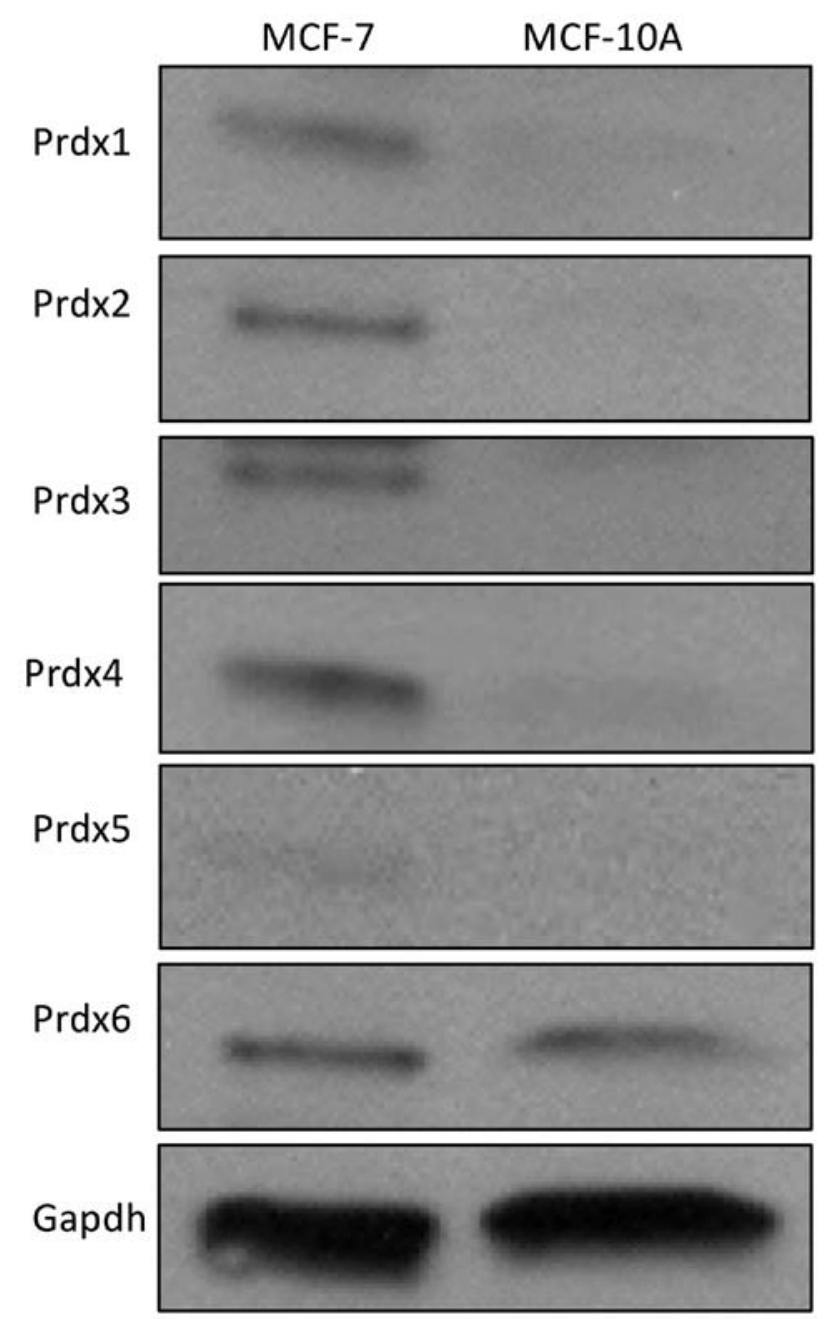

Figure 2. MCF-7 cells express elevated levels of Prdx proteins. MCF-7 and MCF-10A cells were cultured under normal growth conditions. Cells were lysed and Prdx expression measured by western blot analysis. GAPDH expression was used as a loading control.

in either morphology or cytotoxicity between untransfected cells and those transfected with negative control siRNA (data not shown). The only morphological change observed in transfected cells was with Prdx3. While negative-control transfected cells appear to have a normal cobblestone-like appearance (Fig. 4A), Prdx3-transfected cells are significantly smaller and rounder (Fig. 4B). In addition, there were fewer cells in all Prdx3-transfected replicate wells. Likewise, cytotoxicity was significantly increased in Prdx3-transfected cells in the absence of doxorubicin treatment, relative to cells transfected with negative control siRNA (Fig. 4B). No other transfection condition showed an effect. Together, these data show that Prdx3 suppression renders MCF-7 cells more susceptible to death in the absence of doxorubicin, suggesting an important role for this protein in the general viability of these cells.

To examine the effect of Prdx suppression on doxorubicin sensitivity, $48 \mathrm{~h}$ after transfection cells were treated with $0.5 \mu \mathrm{M}$ doxorubicin for $24 \mathrm{~h}$, viable cell number was measured using the MTT assay, which was originally used to compare doxorubicin sensitivity in the cancerous and non-cancerous cell lines. As shown in Fig. 5A, doxorubicin treatment led to a 

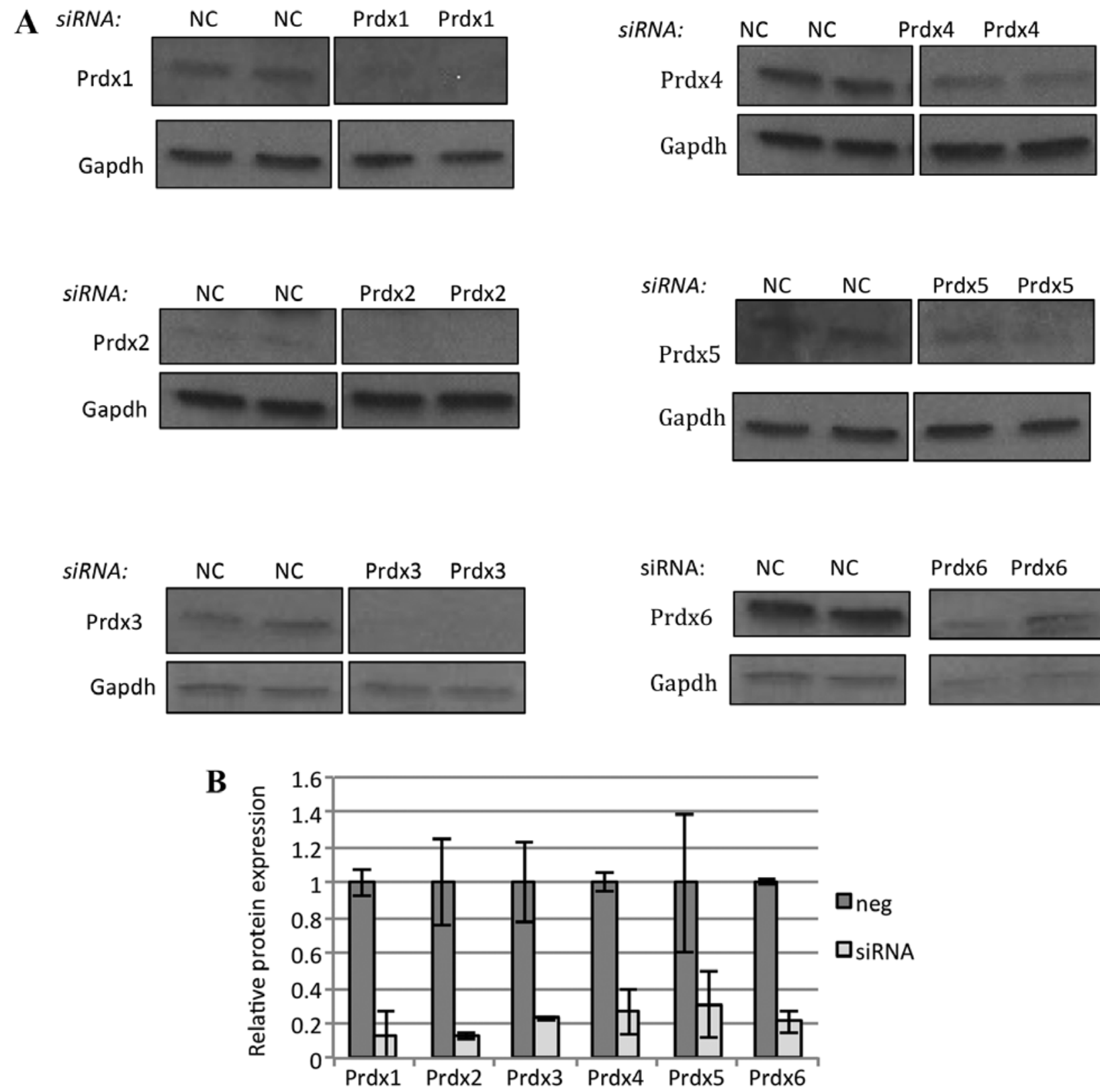

Figure 3. Transient transfection of MCF-7 cells with Prdx siRNA markedly suppresses Prdx expression. MCF-7 cells were transfected with 33 nM negative control siRNA or specific Prdx siRNA and cultured for $48 \mathrm{~h}$. Cells were lysed and Prdx expression measured by western blot analysis. Gapdh expression was used as a loading control. (A) Representative western blots showing expression in replicate transfections. (B) Protein expression was quantified from parallel experiments and normalized to GAPDH levels. Averages from replicate tranfections are shown ( \pm SD).

significant reduction in MTT absorbance in cells transfected with either Prdx1, Prdx2, Prdx3 or Prdx5. The magnitude of this decrease was about $40 \%$ for Prdx1 and Prdx2, and over $50 \%$ for Prdx 3 and Prdx5. These data suggest that reduction in the levels of these Prdx proteins in MCF-7 cells inhibits growth and/or induces death in response to doxorubicin treatment. We attempted to address this by measuring cell death using the Hoechst/Yo-Pro cell staining method. Representative phase contrast and fluorescent images are shown for doxorubicin-treated cells transfected with negative control siRNA, or one of the Prdxs that showed an MTT reduction. A reduced cell number in all Prdx-transfected cells, compared to the negative control, was observed. These data are consistent with the MTT data. Analysis of the stained cells shows a marked increase in the percentage of dead cells in all Prdx-transfected conditions. These data strongly suggest that suppression of Prdx1, Prdx2, Prdx 3 and Prdx 5 in MCF-7 cells increases the susceptibility of MCF-7 cells to doxorubicin-induced cell death.

Since our data suggested a role for Prdxs in doxorubicin resistance in MCF-7 cells, we asked whether long-term treatment of these cells and selection of a highly resistant subline would lead to a concomitant change in Prdx levels. This experiment has important clinical significance since many breast cancers develop resistance with prolonged chemotherapy treatment. Cells were cultured in the presence of $0.1 \mu \mathrm{M}$ doxorubicin for 14 days and Prdx expression analyzed. As shown in Fig. 6A, 14 days of treatment led to a marked increase in the expression of Prdxs 2, 3, 4 and 5. Quantification of these levels 

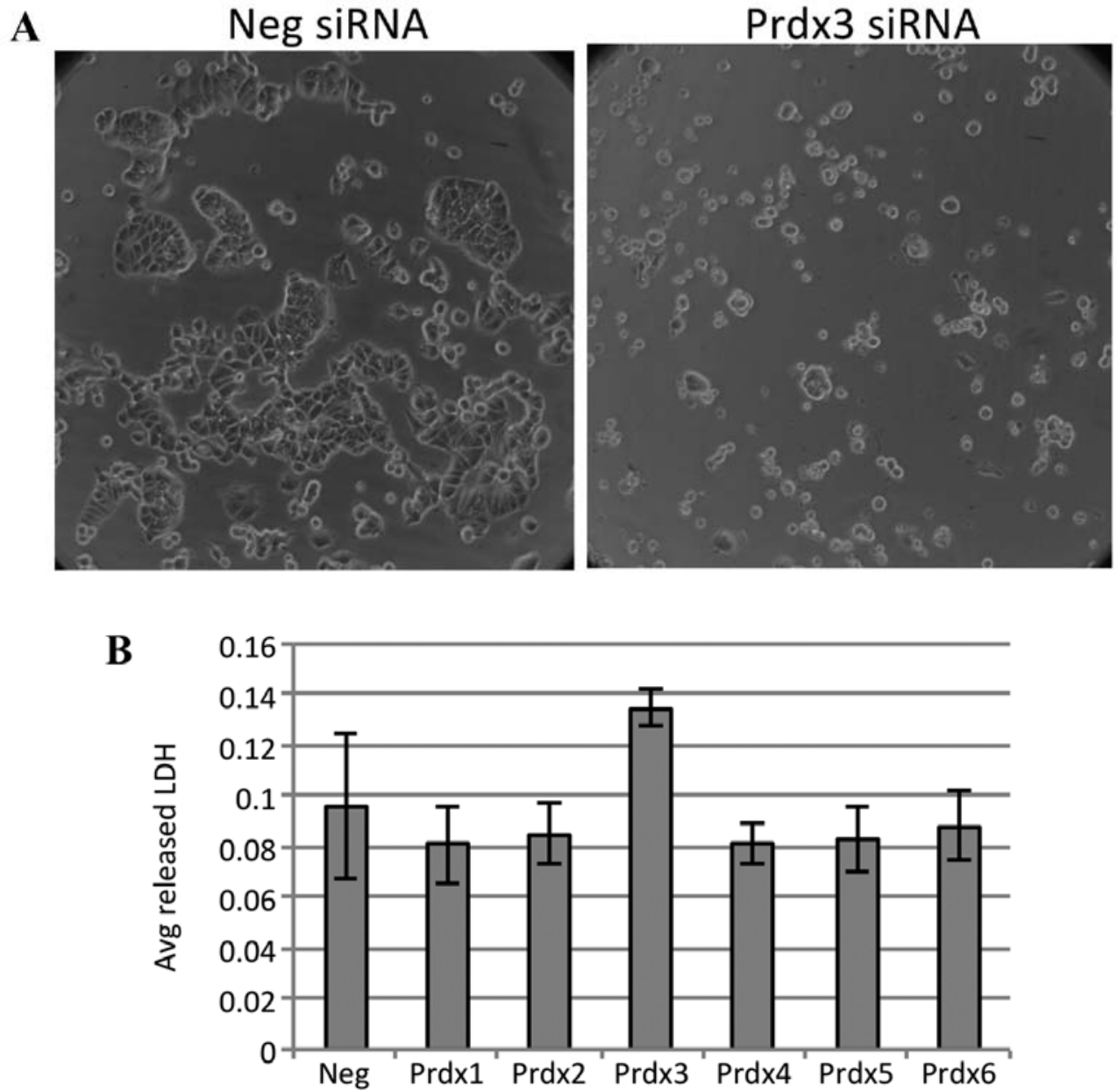

Figure 4. Prdx3 siRNA increases cytotoxicity in MCF-7 Cells. MCF-7 cells were transfected with 33 nM negative control siRNA or specific Prdx siRNA and cultured for $48 \mathrm{~h}$. (A) Cells were photographed with phase contrast microscopy. Representative images for control and Prdx 3 transfected cells are shown. (B) Cytotoxicity was measured using the Released LDH assay. The average of six replicate wells for each treatment are shown ( \pm SD). p $<0.005$ for Prdx3.

are represented in Fig. 6B, revealing a nearly 10X increase in Prdx2 expression, and an approximately 4-fold increase in levels of $\operatorname{Prdx} 3, \operatorname{Prdx} 4$ and $\operatorname{Prdx} 5$. It is clear from these data that culturing MCF-7 cells for 2 weeks in doxorubicin leads to a robust induction of several Prdx proteins.

\section{Discussion}

In the present study, we showed a correlation between doxorubicin-resistance and peroxiredoxin levels between MCF-7 and MCF-10A cells, demonstrating significantly higher resistance and Prdx expression in the cancer line. Using transient transfections of MCF-7 cells with siRNA, we obtained marked reduction in Prdx levels for all six proteins, leading to moderate toxicity in Prdx3-suppressed cells. Subsequent treatment of siRNA-transfected cells with doxorubicin resulted in a reduction in viable cell number with suppression of either Prdx1, $\operatorname{Prdx} 2, \operatorname{Prdx} 3$ and Prdx5. We went on to show that this cell loss was, at least in part, due to apoptotic death. Finally, we demonstrated that 2-week treatment of MCF-7 cells with doxorubicin leads to a marked induction of several Prdx proteins. Together, these data support our hypothesis that Prdxs play a protective role in MCF-7 cells and that doxorubicin-treatment leads to selection of drug-resistant cells that possess elevated Prdx levels.

We and others previously reported the overexpression of Prdxs in MCF-7 cells (22-24), which is consistent with elevated Prdx levels found in breast cancer tissue from patients. However, the mechanism by which these cells upregulate Prdxs is not understood. The Prdx family is inducible by oxidative stress in several systems, and ROS-induced modifications include regulation at both the transcriptional and post-transcriptional levels (4). A previous study from our lab showed that Prdxs1-5 are elevated at the mRNA level in these cells, compared to MCF-10A cells, suggesting a transcriptional mechanism (24), but the signal transduction events and transcription factors mediating higher basal levels are not known. However, there is evidence that Nrf2 coordinately regulates the Prdx gene family in macrophages (28), so we are currently investigating this as a possible mechanism in MCF-7 cells.

Prdx suppression in many cells, including several cancer cell types, is known to increase cell death. Our results suggest a similar protective role for Prdx3 in MCF-7 cells, in the absence of any added oxidative stress. Prdx 3 is a mitochondrial 

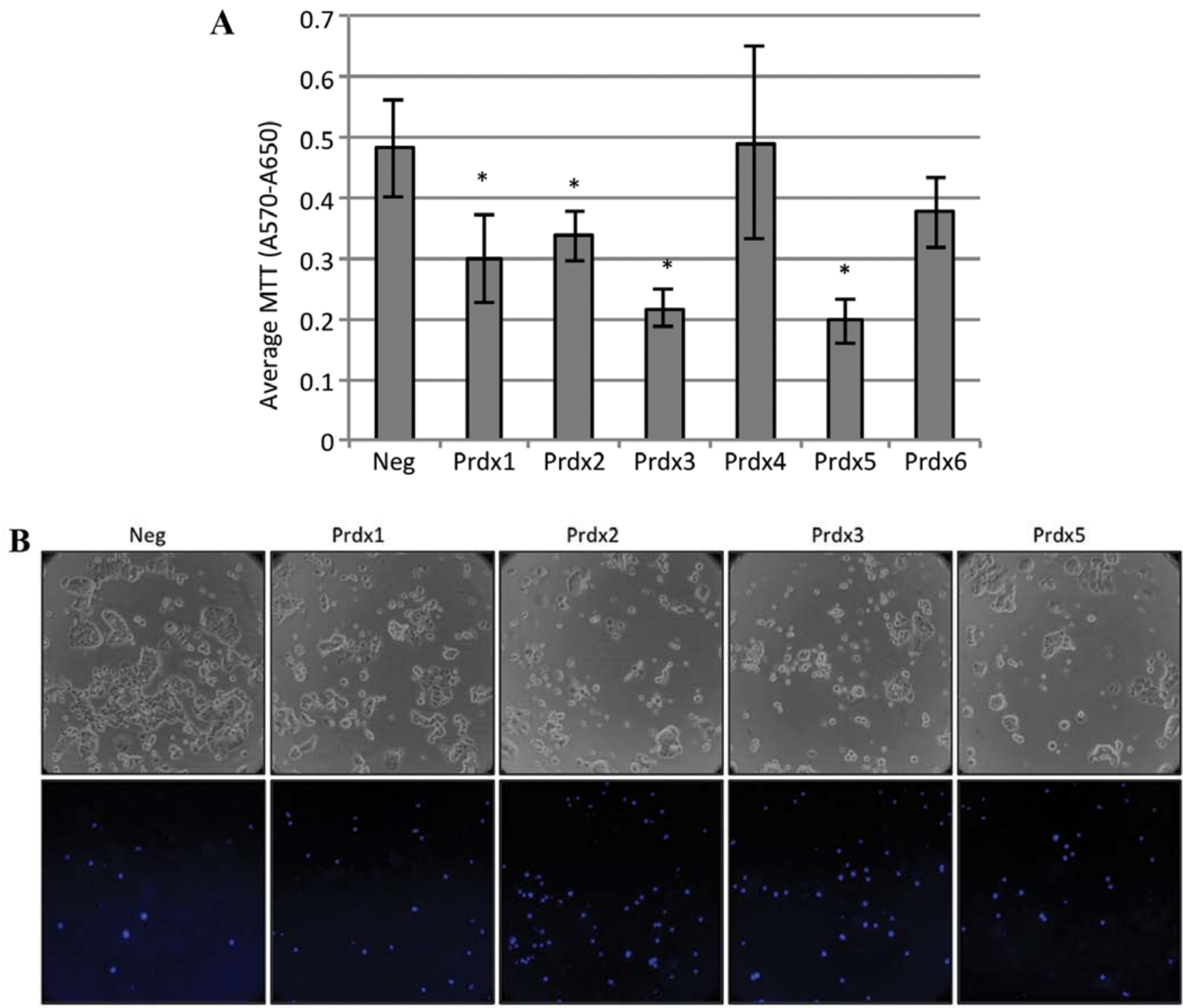

Figure 5. Effect of Prdx suppression on doxorubicin-induced toxicity. MCF-7 cells were transfected with 33 nM negative control siRNA or specific Prdx siRNA, cultured for $48 \mathrm{~h}$, and treated for $24 \mathrm{~h}$ with $0.5 \mu \mathrm{M}$ doxorubicin. (A) Viable cells were quantified using an MTT assay. Formazan absorbance at 570 was measured for each well, with A650 used for background subtraction. Averages of six replicate wells are shown ( \pm SD). "p $<0.005$. (B) Apoptosis was measured in separate wells using Hoechst/Yo-Pro staining and cells were photographed with phase contrast and fluorescence microscopy. Representative images for transfectants showing a significant decrease in MTT levels (Prdx1, Prdx2, Prdx3 and Prdx5) are shown.

peroxiredoxin that is transcriptionally regulated by c-myc and is required for proliferation, transformation, and apoptosis in ovarian cancer cells (29). Recently, a similar function was reported for Prdx3 in cervical cancer cells (30). From these and other studies, the importance of $\operatorname{Prdx} 3$ as a key protective protein in cancer is well established. Our results are the first to demonstrate this same function for Prdx 3 in breast cancer cells, suggesting that this protein may have a more ubiquitous survival function in cancer.

We showed that MCF-7 breast cancer cells are significantly more resistant to doxorubicin-induced toxicity at both 0.1 and $0.5 \mu \mathrm{M}$ concentrations than the non-cancerous MCF-10A cells. Gajewski et al also demonstrated that MCF-10A cells exposed to $0.1 \mu \mathrm{M}$ doxorubicin (a clinically relevant dosage) underwent growth arrest and apoptosis, and also developed elevated levels of ROS (31). However, our demonstration that siRNA-mediated
Prdx suppression markedly increases doxorubicin-induced apoptosis is a novel finding, and one that is consistent with the known ROS-inducing action of doxorubicin as well as the increased susceptibility of Prdx-suppressed MCF-7 cells to ROS-induced apoptosis. For example, Wang et al showed that Prdx2 suppression in MCF-7 cells increased sensitivity to radiation-induced cell death (25), and a recent follow-up study demonstrated that this occurred by alterations in cellular thiol status and intracellular $\mathrm{Ca}^{2+}$ homeostasis (32). Likewise, Prdx1 suppression in MCF-7 cells leads to apoptosis induced by $\beta$-lapachone, an anticancer agent that produces large amounts of ROS induced apoptosis (33). In addition, Bae et al showed that transgenic overexpression of Prdx 1 and Prdx 2 in MCF-10A cells increases their resistance to peroxide-induced cell death (22). Together, there is strong evidence for Prdxs as key protective players against ROS-induced death of breast cancer cells. 

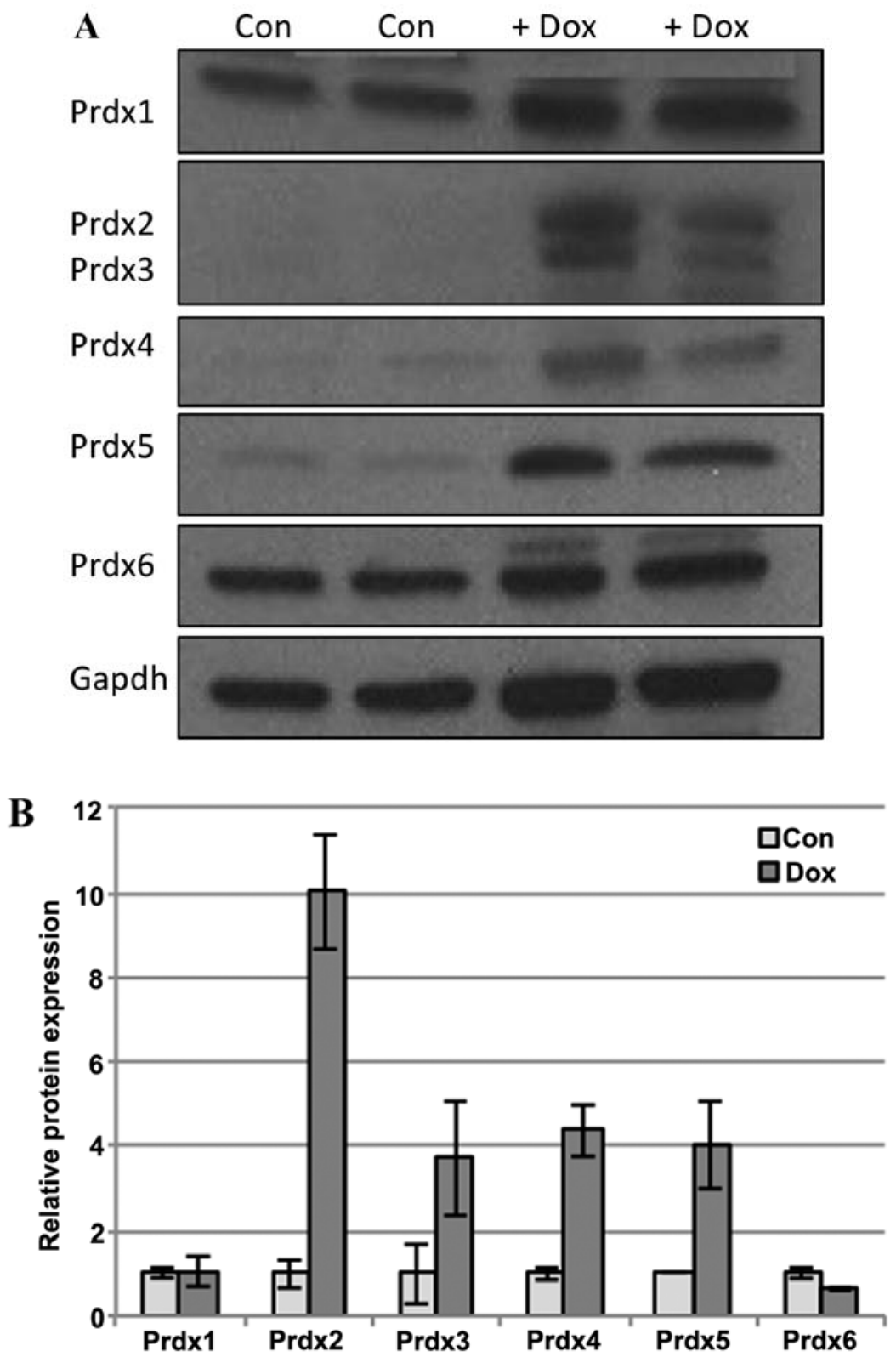

Figure 6. Two-week culture of MCF-7 cells with doxorubicin induces Prdx expression. MCF-7 cells were cultured for 14 days in the presence of $0.1 \mu$ M doxorubicin. Cells from these and control flasks were lysed and analyzed for Prdx expression using western blot analysis. GAPDH expression was used as a loading control. (A) A representative western blot showing expression in replicate treatments. (B) Protein expression was quantified and normalized against GAPDH levels.

Our results further showed a functional relationship between Prdx expression and doxorubicin resistance using a prolonged doxorubicin treatment. The marked induction of several Prdx proteins after a 2-week culture with $0.1 \mu \mathrm{M}$ doxorubicin suggests higher levels in drug-resistant cells. While it is not clear that this Prdx induction is essential for clonal selection of resistant cells, this observation coupled with the data from our transfection experiments strongly suggests an important role for these proteins in cell survival. Interestingly, short-term ( 4 or $24 \mathrm{~h}$ ) treatment of MCF-7 cells with 0.1 or $0.5 \mu \mathrm{M}$ doxorubicin does not alter $\operatorname{Prdx}$ levels (data not shown), suggesting that the changes in gene expression are likely associated with the selection of resistant cells over time.

In conclusion, our data are the first to report an effect of doxorubicin treatment on Prdx expression in breast cancer cells, as well as a protective role for the peroxiredoxin protein family in breast cancer cell resistance to doxorubicin. Since the innate and acquired resistance of many breast tumors to doxorubicin is of critical concern for patients, a better understanding of the mechanisms governing this likely multifactorial phenomenon is essential. While we do not yet understand the precise role of each individual Prdx in the basal antioxidant defense system in these cells, Prdxs may, in fact, play an essential role in the survival of breast cancer cells in vivo. Based on the abundance and obvious importance of this family of antioxidants in normal and cancer cell biology, and the critical role of oxidative stress in chemotherapy success, this area of research warrants further investigation and is likely to provide an important new avenue for new therapeutic interventions for the treatment of breast cancer. 


\section{Acknowledgements}

This study was supported in part by a grant from the Connecticut Breast Health Initiative (CTBHI).

\section{References}

1. Fujii $\mathbf{J}$ and Ikeda $\mathrm{Y}$ : Advances in our understanding of peroxiredoxin, a multifunctional, mammalian redox protein. Redox Rep 7: 123-130, 2002.

2. Hofmann B, Hecht HJ and Flohe L: Peroxiredoxins. Biol Chem 383: 347-364, 2002.

3. Wood ZA, Schröder E, Harris JR and Poole LB: Structure, mechanism and regulation of peroxiredoxins. Trends Biochem Sci 28: 32-40, 2003

4. Immenschuh S and Baumgart-Vogt E: Peroxiredoxins, oxidative stress, and cell proliferation. Antioxid Redox Signal 7: 768-777, 2005 .

5. Rhee SG, Chae HZ and Kim K: Peroxiredoxins: A historical overview and speculative preview of novel mechanisms and emerging concepts in cell signaling. Free Rad Biol Med 38 1543-1552, 2005.

6. Halliwell B and Gutteridge J: Free Radicals in Biology and Medicine. 3rd edition. Oxford University Press, New York, NY 1999.

7. Halliwell B: Oxidative stress and cancer: have we moved forward? Biochem J 401: 1-11, 2007.

8. Valko M, Rhodes CJ, Moncol J, Izakovic M and Mazur M: Free radicals, metals and antioxidants in oxidative stress-induced cancer. Chem Biol Interact 160: 1-40, 2006.

9. Butterfield LH, Merino A, Golub SH and Shau H: From cytoprotection to tumor suppression: the multifactorial role of peroxiredoxins. Antioxid Redox Signal 1: 385-402, 1999.

10. Noh DY, Ahn SJ, Lee RA, Kim SW, Park IA and Chae HZ: Overexpression of peroxiredoxin in human breast cancer. Anticancer Res 21: 2085-2090, 2001.

11. Kinnula VL, Lehtonen S, Sormunen R, Kaarteenaho-Wiik R, Kang SW, Rhee SG and Soini Y: Overexpression of peroxiredoxins I, II, III, V, and VI in malignant mesothelioma. J Pathol 196: 316-323, 2002.

12. Karihtala, P, Mantyniemi A, Kang SW, Kinnula VL and Soini Y: Peroxiredoxins in breast carcinoma. Clin Cancer Res 15: 3418-3424, 2003.

13. Lehtonen ST, Svensk AM, Soini Y, Paakko P, Hirvikoski P, Kang SW and Saily M: Peroxiredoxins, a novel protein family in lung cancer. Int J Cancer 111: 514-521, 2004.

14. Quan C, Cha EJ, Lee HL, Han KH, Lee KM and Kim WJ: Enhanced expression of peroxiredoxin I and VI correlates with development recurrence and progression of human bladder cancer. J Urolol 175: 1512-1516, 2006.

15. Kinnula VL, Paakko P and Soini Y: Antioxidant enzymes and redox regulating thiol proteins in malignancies of human lung. FEBS Lett 569: 1-6, 2004.

16. Li DQ, Wang L, Fei F, Hou YF, Luo JM, Wei-Chen, Zeng R, Wu J, Lu JS, Di GH, Ou ZL and Xia QC: Identification of breast cancer metastasis-associated proteins in an isogenic tumor metastasis model using two-dimensional gel electrophoresis and liquid chromatography-ion trap-mass spectrometry. Proteomics 6: 3352-3368, 2006 .
17. Neumann CA and Fang Q: Are peroxiredoxins tumor suppressors? Curr Opin Pharm 7: 375-380, 2007.

18. Chang XZ, Li DQ, Hou YF, Wu J, Lu JS, Di GH, Jin W, Ou ZL and Shen ZZ: Identification of the functional role of peroxiredoxin 6 in the progression of breast cancer. Breast Cancer Res 9: R76, 2007.

19. Ishii T, Warabi E and Yanagawa T: Novel roles of peroxiredoxins in inflammation, cancer and innate immunity. $\mathrm{J} \mathrm{Clin}$ Biochem Nutr 50: 91-105, 2012.

20. Chahed K, Kabbage M, Hamrita B, Guillier CL, Trimeche M, Remadi S, Ehret-Sabatier L and Chouchane L: Detection of protein alterations in male breast cancer using two dimensional gel electrophoresis and mass spectrometry: The involvement of several pathways in tumorigenesis. Clin Chim Acta 388: 106-114, 2008

21. Coley HM: Mechanisms and strategies to overcome chemotherapy resistance in metastatic breast cancer. Cancer Treat Rev 34: 378-390, 2008

22. Bae J, Ahn S, Han W and Noh D: Peroxiredoxin I and II inhibit $\mathrm{H}_{2} \mathrm{O}_{2}$-induced cell death in MCF-7 Cell Lines. J Cell Biochem 101: 1038-1045, 2007.

23. Goncalves K, Sullivan K and Phelan SA: Differential expression and function of peroxiredoxin 1 and peroxiredoxin 6 in cancerous MCF-7 and noncancerous MCF-10A breast epithelial cells. Cancer Invest 30: 38-47, 2012.

24. Tehan L, Taparra K and Phelan SA: Peroxiredoxin overexpression in MCF-7 breast cancer cells and regulation by cell proliferation and oxidative stress. Cancer Invest 31: 374-384, 2013.

25. Wang T, Tamae D, LeBon T, Shively JE, Yen Y and Li JJ: The role of peroxiredoxin II in radiation-resistant MCF-7 breast cancer cells. Cancer Res 65: 10338-10346, 2005.

26. Hickman JA: Apoptosis induced by anticancer drugs. Cancer Metastasis Rev 11: 121-139, 1992.

27. Osbild S, Brault L, Battaglia E and Bagrel D: Resistance to cisplatin and adriamycin is associated with the inhibition of glutathione efflux in MCF-7-derived cells. Anticancer Res 26: 3595-3600, 2006.

28. Ishii T, Itoh K, Takahashi S, Sato H, Yanagawa T, Katoh Y, Bannai S and Yamamoto M: Transcription factor Nrf2 coordinately regulates a group of oxidative stress-inducible genes in macrophages. J Biol Chem 275: 16023-16029, 2000.

29. Wonsey DR, Zeller KI and Dang CV: The c-Myc target gene PRDX3 is required for mitochondrial homeostasis and neoplastic transformation. Proc Natl Acad Sci USA 99: 6649-6654, 2002

30. Li L, Zhang YG and Chen CL: Anti-apoptotic role of peroxiredoxin III in cervical cancer cells. FEBS Open Bio 3: 51-54, 2012.

31. Gajewski E, Gaur S, Akman SA, Matsumoto L, van Balgooy JNA and Doroshowa JH: Oxidative DNA base damage in MCF-10A breast epithelial cells at clinically achievable concentrations of doxorubicin. Biochem Pharmacol 73: 1947-1956, 2007.

32. Diaz AJG, Tamae D, Yen Y, Li JJ and Wang T: Enhanced radiation response in radioresistant MCF-7 cells by targeting peroxiredoxin II. Breast Cancer (Dove Med Press) 5: 87-101, 2013.

33. He T, Banach-Latapy A, Vernis L, Dardalhon M, Chanet R and Huang ME: Peroxiredoxin 1 knockdown potentiates $\beta$-lapachone cytotoxicity through modulation of reactive oxygen species and mitogen-activated protein kinase signals. Carcinogenesis 34: 760-769, 2013. 